Z Herz-Thorax- Gefäßchir 2009 · 23:330-330

DOI 10.1007/s00398-009-0752-7

Online publiziert: 4. Dezember 2009

(c) Springer-Verlag 2009

\author{
H.-R. Zerkowski \\ Managing Editor HTG, Riehen
}

\title{
Der Schlüssel künftigen Erfolgs
}

„Interdisziplinäres Arbeiten ist der Schlüssel zu künftigem beruflichem Erfolg" auch innerhalb unseres Fachgebiets. Dessen Zukunft dürfte - gegründet auf solidem chirurgischem Handwerk, sauber erarbeiteter Evidenz, Gespür, Begeisterung und Empathie - so schlecht nicht sein.

So oder zumindest sinngemäß formulierte es unlängst Jens Michael Polonius, einer der Altvorderen (auch wenn man es ihm nicht anmerkt oder gar ansieht) unseres Fachs, auf dem 16. (!) Facharztseminar von DGTHG, DGT und BDC in Mainz.

Wir verfolgen die Richtung der gehobenen, den Blick öffnenden Fort- und Weiterbildung. Und nachdem wir auch im zweiten Jahr der Zeitschrift im neuen Gewand das Kaleidoskop des Alltags der Herz-, Thorax- und Gefäßchirurgen erfolgreich in Weiterbildungsinhalte übersetzen konnten, gehen wir nun bereits ins dritte Jahr.

Auch heute und mit dieser Ausgabe gibt es also wieder Hilfe zur Selbsthilfe: das Angebot umfasst Beiträge zur chirurgischen Therapie der akuten Typ-A-Dissektion sowie zur Stammzelltherapie der Herzinsuffizienz, die einen Blick über den Tellerrand ermöglichen, der staunend zeigt, dass auch noch anderes direkt auf unserem Tisch liegt. Und auch die Artikel zum Begriff des „off-label use“, der Krankenhausfinanzierung eines Universitätskrankenhauses und erstmals eine leitliniennahe Publikation aus einer Arbeitsgruppe, hier zu Herzchirurgie-assoziierten Nierenfunktionsstörungen, bieten hervorragende Informationen.

Es scheint mir, die Zeitschrift Herz-, Thorax- und Gefäßchirurgie ist ,erwachsen" geworden.
Viel Spaß - und lassen Sie uns wissen, ob die Richtung noch stimmt; per E-Mail an die Herausgeber, als Leserbrief an die Redaktion oder - am allerliebsten - als Beitrag für die Zeitschrift.

Ihr

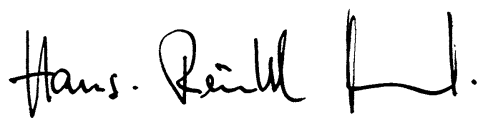

H.-R. Zerkowski

\section{Korrespondenzadresse}

Prof. Dr. H.-R. Zerkowski

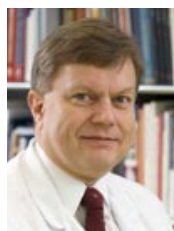

Managing Editor HTG

Bahnhofstr. 25

4125 Riehen

Schweiz

zerkowski.htg@bluewin.ch 